REVIEW

\title{
Multiple sclerosis: diagnosis and the management of acute relapses
}

\author{
S M Leary, B Porter, A J Thompson
}

Postgrad Med J 2005;81:302-308. doi: 10.1136/pgmj.2004.029413

Multiple sclerosis is an inflammatory demyelinating disease of the central nervous system that may result in a wide range of neurological symptoms and accumulating disability. Its course is unpredictable resulting in a changing pattern of clinical need. Diagnostic criteria for multiple sclerosis require objective evidence for dissemination in space and time. The diagnostic and management process should follow good practice guidelines with the person at the centre of the process. Appropriate support and information should be available from the time of diagnosis. Continuing education is key in enabling the person to actively participate in their management. In the event of an acute relapse the person should have direct access to the most appropriate local service. Provided medical causes have been excluded, corticosteroid treatment to hasten the recovery from the relapse should be considered. Management of an acute relapse should be comprehensive addressing any medical, functional, or psychosocial sequelae.

See end of article for authors' affiliations

......

Correspondence to: Professor A J Thompson, Rehabilitation Group, Institute of Neurology, Queen Square, London WCIN 3BG, UK; a.thompson@ion.ucl.ac.uk

Submitted 13October 2004 Accepted

16 November 2004
M ultiple sclerosis affects about 85000 people in the United Kingdom and is the most common cause of neurological disability in young adults. The National Institute of Clinical Excellence (NICE) has recently published management guidelines that highlight the need to improve standards of care offered to people with this condition. ${ }^{1}$ Health and social care professionals in both primary and secondary care should be informed of the current evidence based practice to optimise their management of people with multiple sclerosis. People with multiple sclerosis must participate fully in decisions on their care. ${ }^{2}$ This review covers both the diagnosis of multiple sclerosis and the management of acute relapses and begins with a brief overview of the condition.

\section{OVERVIEW OF MULTIPLE SCLEROSIS}

Multiple sclerosis is characterised by inflammatory, demyelinating lesions scattered throughout different sites and at different times. The exact pathophysiology of multiple sclerosis is not clear but it seems to be autoimmune in nature. The wide distribution of lesions results in a variety of clinical features such as loss of sensation, muscle weakness, visual loss, incoordination, cognitive the central nervous system (CNS) that occur at impairment, fatigue, pain, and bladder and bowel disturbance. The course of multiple sclerosis is unpredictable resulting in a changing pattern of need over time. It has comparatively little effect on life expectancy and therefore usually has to be managed for many decades. ${ }^{3}$

Multiple sclerosis typically presents between 20 and 40 years of age. Women are more susceptible than men by a factor that approaches $2: 1$. Race and geography also affect susceptibility to the disease. ${ }^{4}$ As a general rule, although with clear exceptions, the risk of multiple sclerosis increases with increased distance from the equator. Migration studies have also suggested that environmental factors in childhood may have an aetiological role. ${ }^{5}$

\section{TYPES OF MULTIPLE SCLEROSIS}

The natural course of multiple sclerosis is extremely variable with symptoms that may appear, disappear, reappear, or gradually worsen over time. Despite this variability it is possible to recognise broad categories of disease and to classify people according to the course of their disease. $^{6}$

\section{Relapsing remitting multiple sclerosis (RRMS)}

This is the most common type of multiple sclerosis; about $85 \%$ of people present with this pattern. RRMS presents with acute or sub-acute onset of neurological symptoms, from which people may recover either completely or partially. Further relapses may then occur at irregular intervals. If recovery from relapses is incomplete people may accrue neurological deficit and disability. A recent study has highlighted the effect of relapses on development of residual deficit; a measurable and sustained increase in disability was seen in up to $42 \%$ of patients at follow up after a relapse. ${ }^{7}$

\section{Secondary progressive multiple sclerosis (SPMS)}

Over time RRMS may convert to a disease pattern of gradual progression with accumulating irreversible neurological deficit and disability (SPMS). The proportion of people developing progressive disease increases with length of follow up. In a Canadian study $41 \%$ of people with RRMS entered the secondary progressive phase within 6-10 years of disease onset increasing to $58 \%$ between 11 and 15 years after onset. ${ }^{8}$ People with SPMS may continue to have superimposed relapses. 
Primary progressive multiple sclerosis (PPMS)

PPMS is characterised by progressive disease from onset, with a gradual accumulation of neurological deficit or disability, without relapse or remission and accounts for about $10 \%-15 \%$ of multiple sclerosis. The average age of onset, about 40 years, is later than in RRMS and comparatively more men are affected resulting in an equal male: female ratio. ${ }^{9}$ The age of onset and rate of progression is similar to the progressive phase of SPMS. ${ }^{10}$

\section{Progressive relapsing multiple sclerosis (PRMS)}

PRMS is defined as progressive disease from onset with superimposed relapses. The progressive disease is predominant and PRMS is considered to be largely similar to PPMS. ${ }^{11}{ }^{12}$ About $10 \%-15 \%$ of people with PPMS will have superimposed relapses.

\section{DISEASE ACTIVITY}

The activity of disease in multiple sclerosis is also extremely variable ranging from clinically asymptomatic, with only pathological evidence of disease detected incidentally at postmortem examination, to an aggressive course with rapidly accumulating disability. An aggressive or malignant disease course may result from frequent relapses with little or no neurological recovery or from rapid disease progression. Benign multiple sclerosis refers to a disease course with minimal or no disability many years after disease onset. The proportion of people with benign disease is a minority and decreases with length of follow up. For example, in an Irish study $42 \%$ of people had benign disease after 10 years but this had decreased to $34 \%$ after 15 years. ${ }^{13}$ Although it is recognised that longstanding benign disease can become more aggressive and lead on to severe disability, ${ }^{8}$ the longer the duration of multiple sclerosis and the lower the disability, the more likely a person is to remain stable. ${ }^{14}$

\section{DIAGNOSIS OF MULTIPLE SCLEROSIS Diagnostic criteria}

Multiple sclerosis remains a clinical diagnosis requiring appropriate expertise to confirm evidence of CNS lesions disseminated in time and space and to exclude other diseases that may give a similar picture. Diagnostic criteria have been developed to improve the certainty of diagnosis. In 1965 Schumacher et al deemed six criteria to be essential in diagnosing multiple sclerosis (box 1 ). ${ }^{15}$

Box 1 Diagnostic criteria according to Schumacher ef al $1965^{15}$

- Age of onset within 10 to 50 years

- Objective neurological signs present on examination

- Neurological symptoms and signs indicative of CNS white matter disease

- Dissemination in space:

- two or more non-contiguous anatomical areas involved

- Dissemination in time:

- two or more episodes of worsening lasting at least 24 hours separated by one month or more, or

- progression over at least six months

- No better explanation by a physician competent in neurology
According to the number of criteria satisfied, the diagnosis was classified as clinically definite, probable, or possible multiple sclerosis. Forty years later the clinical principles of these criteria remain valid although modifications have been recommended reflecting the development of diagnostic investigations. In 1983 Poser et al modified the criteria to incorporate paraclinical and laboratory evidence and to extend the age of onset to 59 years. ${ }^{16}$ These criteria classified multiple sclerosis as clinically or laboratory supported definite/probable based on clinical and paraclinical evidence of disseminated lesions and oligoclonal bands in the cerebrospinal fluid (CSF).

The diagnostic criteria have been further revised by McDonald et al in 2001. ${ }^{17}$ These criteria still place an emphasis on dissemination of lesions in time and space and incorporate both clinical and paraclinical evidence, notably magnetic resonance imaging (MRI), which is recognised as the most sensitive and specific paraclincal test. MRI criteria have been defined for both dissemination in space and time. Diagnostic criteria have been recommended for different clinical presentations (box 2). If the criteria are fulfilled, the diagnosis is "multiple sclerosis"; if the criteria are not completely fulfilled, the diagnosis is "possible multiple sclerosis"; if the criteria are fully explored and not fulfilled, the diagnosis is "not multiple sclerosis". The terms clinically and laboratory supported definite/probable multiple sclerosis are no longer recommended.

MRI is the investigation of choice but visual evoked potential studies may also provide diagnostic support. Examination of CSF is only required if there is clinical uncertainty. If there are atypical clinical features further investigations may be required to exclude other diseases.

\section{DIFFICULT DIAGNOSTIC GROUPS}

Diagnosis may be straightforward when a person presents having had two discrete neurological episodes suggestive of multiple sclerosis disseminated in space and time with objective evidence on examination. However, people may present in a number of ways that cause difficulty in diagnosis. Some examples are now discussed.

\section{Monosymptomatic presentation}

It is common for people to present after their first episode of neurological symptoms, such as an optic neuritis or a sensory cord episode, with no clinical evidence of dissemination. The revised diagnostic criteria have included criteria for such monosymptomatic presentations. Dissemination in space may often be evident on MRI at initial presentation but dissemination in time cannot be proved if MRI is performed within three months of presentation. It is suggested that evidence for dissemination in time may be provided if criteria are fulfilled on delayed or serial MRI. However, if the MRI at presentation shows multiple typical lesions, a more pragmatic approach is to discuss with the person that the clinical and investigation findings are in keeping with possible multiple sclerosis and if there is a second clinical episode the diagnosis of multiple sclerosis will be confirmed.

Thorough history taking is also important in this situation. During the diagnostic stage it is not unusual for people to recall having had mild neurological symptoms in the past. Such episodes may have not been reported previously as they have recovered quickly or were deemed too trivial to report to a doctor. The significance of previous symptoms has to be evaluated on an individual basis; transient sensory symptoms are not uncommon in the general population but an episode of Lhermittes phenomenon may be highly suggestive of a previous demyelinating episode. Although historical reports are not objective evidence they may raise the suspicion of the diagnosis. 
Box 2 Diagnostic tests required for different presentations of multiple sclerosis according to McDonald et al 2001 (detailed criteria for MRI abnormality are defined but are not given here) ${ }^{17}$

Two or more episodes; objective clinical evidence of two or more lesions

- No additional tests required

Two or more episodes; objective clinical evidence of one lesion

- Dissemination in space shown by

- MRI (nine or more T2 lesions or one gadolinium enhancing lesion), or equivocal MRI (two or more lesions) plus positive CSF, or

- await further clinical episode implicating a different site

One episode; objective clinical evidence of two or more lesions

- Dissemination in time shown by

- MRI, or second clinical episode

One episode; objective clinical evidence of one lesion (monosymptomatic presentation)

- Dissemination in space shown by

- MRI, or equivocal MRI plus positive CSF, and

- Dissemination in time, shown by

- MRI, or second clinical episode

Insidious neurological progression suggestive of MS

- Positive CSF, and

- Dissemination in space, shown by

- MRI, or abnormal VEP associated with equivocal $M R I$, and

- Dissemination in time, shown by

- MRI, or continued progression for one year

\section{Insidious neurological progression (PPMS)}

The onset of PPMS is insidious and there may be a long delay from disease onset to diagnosis. Most people with PPMS present with a single progressive symptom, usually a paraparesis implicating the spinal cord and so alternative diagnoses such as spinal cord compression need to be excluded. ${ }^{18} 19$ Diagnosis of PPMS has historically been hampered by the lack of specific diagnostic criteria but these have now been developed. ${ }^{17} 20 \mathrm{~A}$ diagnosis of PPMS cannot be made on the clinical features alone and more emphasis is put on paraclinical evidence. In particular a definite diagnosis of PPMS cannot be made without showing the presence of oligoclonal bands in the CSF that are not present in paired serum.

\section{Explosive onset}

Multiple sclerosis may occasionally have an explosive onset with clinical involvement of multiple areas of the CNS at first presentation. This may be difficult to differentiate from acute disseminated encephalomyelitis (ADEM), a monophasic demyelinating disease of the CNS. ${ }^{21}$ ADEM classically occurs after an infection or vaccination but there may be no preceding history and multiple sclerosis may similarly present after an immune trigger. ADEM may be more likely to be associated with headache, fever, and drowsiness but encephalopathic presentations of multiple sclerosis also occur especially in children. ${ }^{22}$

MRI in ADEM typically shows extensive multifocal white matter abnormalities but the appearances cannot be reliably differentiated from multiple sclerosis. However, serial MRI showing new lesions is in favour of a diagnosis of multiple sclerosis. ${ }^{23}$ CSF examination does not reliably distinguish the diseases and oligoclonal bands may be present in ADEM.

Although establishing a definite diagnosis may not be possible at presentation first line treatment of the acute episode is the same in both diseases, namely corticosteroids. The diagnosis may become clear with time if the person has a second episode suggestive of multiple sclerosis and this possibility should be discussed with the person.

\section{Vague symptoms}

A not uncommon scenario is that a person presents with a history of vague or mild neurological symptoms, often sensory, and is concerned that they have multiple sclerosis. Even if the history is not particularly suggestive of multiple sclerosis and neurological examination is normal, without an alternative explanation for the symptoms the possibility of multiple sclerosis cannot be definitely excluded. There are no clear guidelines for this situation but the decision as to whether to investigate should be made in discussion with the person. If a decision is made against investigation and the person does later develop multiple sclerosis the delay in diagnosis is unlikely to change long term outcome. However, if a diagnosis of multiple sclerosis is suspected most people would favour knowing the diagnosis. ${ }^{24}$

\section{INVOLVING THE PERSON IN THE DIAGNOSTIC PROCESS}

People with multiple sclerosis attach great importance to involvement in and clear communication throughout the diagnostic process. The diagnostic phase has been described as a time of anxious waiting. ${ }^{25}$ In particular the delay between presenting symptoms, diagnostic tests and receipt of test results is a recurring area of complaint. ${ }^{26} 27$

People with multiple sclerosis want a clear accurate diagnosis, access to appropriate support, information, and continuing education at and around the time of diagnosis. ${ }^{28}$ The NICE guidelines on multiple sclerosis mirror these principles making a number of recommendations of good practice (box 3).

\section{DIAGNOSTIC MODELS - GETTING IT RIGHT FROM THE START}

Despite national circulation of guidance documents on standards of care, ${ }^{128}$ there is currently a wide variation and lack of equity in the services provided throughout the United Kingdom and diagnosis often occurs in a haphazard and unsupported way. This situation may be partly explained by the fact that medical and neurology services are often oversubscribed with long waiting lists for clinics and investigations. There is some evidence that a coordinated diagnostic clinic model facilitates an effective, supportive, efficient, and cost effective structure to manage the diagnostic phase of multiple sclerosis. ${ }^{29}$ Such diagnostic models serve as an illustration of how reorganisation, flexibility, team working, and forward planning can influence the delivery of care. However, outside of specialist centres the model that is chosen for a diagnostic service will depend on the expertise and resources available locally. 
Box 3 The diagnostic process. Management of multiple sclerosis in primary and secondary care, NICE 2003

- A person who is suspected of having MS should be referred to a specialist neurology service, and seen rapidly within an audited time (will vary according to clinical need but should be no longer than six weeks).

- A person should be informed of the potential diagnosis as soon as a diagnosis of MS is considered probable, before undertaking further investigations.

- Throughout the diagnostic process, the healthcare professional should:

- find out what and how much information the person wants to receive;

- discuss the nature and purpose of all investigations.

- The person should be seen again after all investigations have been completed (recommended within a further six weeks) and the diagnosis confirmed or refuted. If the diagnosis is confirmed the person should be told the diagnosis by a doctor with specialist knowledge of MS; this will usually be a consultant or an experienced specialist registrar.

- After diagnosis the person should be:

- offered at least one more appointment in the near future (recommended no longer than four weeks) to see wherever possible the doctor who gave the diagnosis;

- put in touch with a skilled nurse or support worker, ideally with specialist knowledge of MS;

- offered written information about disease specific support organisations;

- offered information about the disease specific to the newly diagnosed.

- Within six months of diagnosis, the person should be offered the opportunity to participate in an educational programme to cover all aspects of MS.

\section{COMMUNICATION, INFORMATION, AND SUPPORT}

People with multiple sclerosis want to be involved in their overall management plan. There is evidence that the quality of communication at the time of diagnosing a chronic disease influences patient health outcomes. ${ }^{30}$ Good history taking and discussion of management plans have been found to have positive effects on emotional health, symptom resolution, general functioning, physiological measures, and pain control. ${ }^{30}$

In addition to good communication people with multiple sclerosis require timely, accurate information and support. Within the United Kingdom multiple sclerosis specialist nurses play a vital part in this area offering one to one educational and supportive sessions. ${ }^{31}$ Educational programmes including "Getting to Grips" courses for the newly diagnosed, and specific workshops for people with secondary or primary progressive disease are also coordinated across the United Kingdom by the Multiple Sclerosis Society.

Multiple sclerosis healthcare professionals also work closely with the voluntary sector to produce educational materials that vary from information booklets, to teaching manuals to help explain the biology of multiple sclerosis, ${ }^{32}$ to online decision making aids that help people choose drug treatments. $^{33}$ This collaborative approach to management reflects the principles of the NHS expert patient programmes that encourage optimal self management strategies. ${ }^{34}$

\section{MANAGEMENT OF ACUTE RELAPSES}

An acute relapse refers to an episode of neurological disturbance, of the kind seen in multiple sclerosis, that lasts for at least 24 hours, and for which there is no other cause such as fever. ${ }^{17}$ Typically a relapse evolves over a few days, reaches a plateau, and then remits to a variable degree over a few weeks or months. The patient experiencing a relapse has to cope with a comparatively sudden onset of neurological symptoms that may be physically and psychologically distressing and functionally and socially incapacitating. In the longer term, incomplete remission from a relapse may result in residual neurological deficit. Management of an acute relapse requires a comprehensive approach addressing its medical, functional, and psychosocial effects. Management incorporates education regarding relapses, support in the event of a relapse, treatment to accelerate or improve the recovery from a relapse, and symptomatic treatment and rehabilitation. The other area of management is to reduce the frequency of relapses with disease modifying therapies such as interferon beta. Disease modifying therapies are not discussed here but have been reviewed extensively elsewhere. ${ }^{35} 36$

\section{Education and support}

Education and support are an ongoing part of management from diagnosis. As discussed earlier people with multiple sclerosis should be provided with information regarding the disease and its management to enable them to actively participate in their own management. In terms of relapses, people should be given information regarding general health factors, such as infection, that may influence the risk of relapse and advised what to do in the event of new symptoms suggestive of a relapse, including how to self refer into primary or secondary care clinics.

\section{Factors affecting relapse activity}

Several general health factors have been proposed to affect relapse activity in multiple sclerosis. Infections may trigger a relapse or exacerbate existing symptoms. Where possible infections should be anticipated and treated early. An association has been reported between common viral infections and relapse but these are difficult to avoid. ${ }^{37-39}$ Infections may occur as a complication of multiple sclerosis, for example urinary tract infections due to retention, and chest infections due to aspiration, so addressing their underlying causes may be preventative.

The possibility that vaccinations may immunologically trigger a relapse has been raised but there is no clear evidence that this is the case. Most vaccines have not been studied prospectively but a double blind, placebo controlled trial of influenza vaccine found no effect on relapse rate or disease progression..$^{40}$ Particular concern has been expressed regarding hepatitis B vaccination but a large case-crossover study found no increase in risk of relapse after hepatitis B or any other vaccination..$^{41}$ Current consensus is that vaccinations are not contraindicated in multiple sclerosis and people with multiple sclerosis should be offered vaccination against influenza. ${ }^{142}$ However, it should not be forgotten that live vaccines may be contraindicated in people receiving immunosuppressant therapy.

Physical trauma has also been proposed to trigger relapses but a thorough review of the evidence a few years ago did not support a link between physical trauma and relapses. ${ }^{43}$ The relation between psychological stress and relapses has been unclear, ${ }^{43}{ }^{44}$ but a recent prospective study found stressful life events were associated with an increased risk of relapse. ${ }^{45} \mathrm{~A}$ recent meta-analysis also supported an association between 
stressful life events and subsequent relapses although did not link specific stressors to relapses. ${ }^{46}$

The effect of pregnancy on disease activity is an important concern among young women. A prospective study of pregnancy in multiple sclerosis confirmed that relapse rate declines during pregnancy, especially in the third trimester, increases during the first three months post-partum, and then returns to pre-pregnancy rate. ${ }^{47}$ In the same study epidural analgesia and breast feeding did not increase the risk of relapse. There is therefore no medical contraindication to pregnancy in multiple sclerosis.

\section{Assessment of new or increased symptoms}

In the event of new or increased symptoms people with multiple sclerosis should be able to identify and contact a professional from their healthcare team who can advise them or direct them to the most appropriate local service. The NICE guidelines recommend that if a person with multiple sclerosis develops new or increased neurological symptoms a formal assessment should be made to determine the diagnosis (box 4).

At the assessment the possibility of any other medical cause for an increase in neurological symptoms must be considered. In particular it is important to exclude an infective cause such as a urinary tract infection, which may be otherwise clinically silent. The possibility of dual neurological pathology, for example, a cord compression mimicking a spinal cord relapse, should also be borne in mind. If the new symptoms are assessed to be unrelated to multiple sclerosis it must still be ensured that the person with multiple sclerosis has access to the appropriate service and treatment.

In developing a service for people with multiple sclerosis it is of key importance that the service is responsive and flexible and so can meet the unpredictable and acute needs of people with multiple sclerosis. As there are often long waiting lists for general neurology/medical clinics this may be difficult. One model to tackle this problem is a specialist multiple sclerosis relapse clinic, which is specifically set up to respond to and assess and manage acute episodes.

\section{Treatment of acute relapses}

If an acute episode of neurological symptoms is assessed to be a relapse of multiple sclerosis treatment to hasten the recovery from the relapse should be considered. The only recommended treatment is corticosteroid therapy. NICE guidelines recommend that corticosteroid therapy should be offered if the episode is sufficient to cause distressing symptoms or an increased limitation on activities.

\section{Box 4 Assessment of new or increased} symptoms. Management of multiple sclerosis in primary and secondary care, NICE $2003^{1}$

- If a person has a comparatively sudden increase in neurological symptoms or disability, or develops new neurological symptoms, a formal assessment should be made to determine the diagnosis (reason for change).

- This diagnostic assessment should:

- Be undertaken within a time appropriate to the clinical presentation;

- Consider the presence of an acute infective cause;

- Involve a GP or acute medical/neurological services.

- Further neurological investigation should not be undertaken unless the diagnosis of MS itself is in doubt.
Evidence base for corticosteroid therapy

There is good evidence that corticosteroid therapy accelerates the recovery from relapse. The exact mode of action of corticosteroids in multiple sclerosis is unclear. However, there are several potential modes of action, which include reducing oedema, stabilising the blood-brain barrier, decreasing proinflammatory cytokines, and inducing $\mathrm{T}$ cell apoptosis. ${ }^{48}$ Efficacy was shown in early studies using intramuscular ACTH though this practice has now stopped. Intravenous methylprednisolone has been shown to accelerate recovery rate, ${ }^{49}{ }^{50}$ and to be effective as ACTH. ${ }^{51} 52$

The comparative efficacy of intravenous and oral corticosteroids has been more controversial. The largest study to date was a randomised, placebo controlled study of intravenous methylprednisolone, followed by oral prednisone, and oral prednisone alone, which was carried out in acute optic neuritis. ${ }^{53}$ In the intravenous corticosteroid group visual recovery was accelerated but there was no long term benefit to vision. In the oral prednisone group there was no improvement in visual outcome and there was an increased rate of new episodes of optic neuritis. At two years there was a reduction in the rate of development of multiple sclerosis in the intravenous group but this was not sustained at the three year follow up. ${ }^{54}$ Other studies have shown no clear advantage of intravenous over oral methylprednisolone in acute relapses. ${ }^{556}$ The choice of corticosteroid therapy remains variable among neurologists although the most popular regimen is an infusion of one gram daily over three days. ${ }^{57}$ NICE guidelines recommend either intravenous methylprednisolone, $500 \mathrm{mg}-1 \mathrm{~g}$ daily, or high dose oral methylprednisolone, $500 \mathrm{mg}-2 \mathrm{~g}$ daily, for between three and five days. To date corticosteroid therapy has only been definitively proved to hasten the recovery from relapses. The effect on long term outcome has not been established. ${ }^{58}$

There has been debate as to the optimal location for the administration of intravenous corticosteroid therapy. Intravenous corticosteroids may be given as a hospital inpatient, in an outpatient clinic, or at home. Factors that may influence the choice of location include cost effectiveness, nursing dependency, and patient preference. In a Canadian study both outpatient and at home treatment were found to be more cost effective than in-patient treatment. ${ }^{59}$ A study of outpatient compared with home treatment in the United Kingdom is currently underway. ${ }^{60}$

\section{Risks of corticosteroid therapy}

The potential benefits and risks should be explained to a person when considering corticosteroid therapy. It must be emphasised that corticosteroids do have side effects although their incidence is comparatively low. In the short term, complications from intravenous methylprednisolone include disturbance of taste, facial flushing, insomnia, psychiatric disturbance, exacerbation of acne and transient hyperglycaemia, and hypertension. ${ }^{61}$ Infection may be exacerbated and should be excluded before starting corticosteroid therapy; urine analysis to exclude a urinary tract infection should be performed routinely. Gastrointestinal disturbance, such as peptic ulceration, may be exacerbated and it is sensible to screen for a history of other risk factors such as use of nonsteroidal anti-inflammatory drugs or excess alcohol intake. The long term complications of corticosteroid therapy are generally not a problem with intermittent courses of intravenous corticosteroids but may be more likely to occur with oral corticosteroids that are more susceptible to misuse. Aseptic bone necrosis has been reported rarely. There is no clear evidence on how often it is acceptable to treat with intravenous corticosteroids but it is recommended not to have more than three courses a year. ${ }^{1}$ 


\section{Key references}

- National Institute for Clinical Excellence. Management of multiple sclerosis in primary and secondary care. London: NICE, 2003.

- McDonald WI, Compston A, Edan G, et al. Recommended diagnostic criteria for multiple sclerosis: guidelines from the international panel on the diagnosis of multiple sclerosis. Ann Neurol 2001;50:1217.

- Medicines Partnership. Multiple sclerosis decisions. An independent aid to your decision. http://www.msdecisions.org.uk.

- Filippini G, Brusaferri F, Sibley WA, et al. Corticosteroids for acute exacerbations in multiple sclerosis. Cochrane Library. Issue 4. Oxford: Update Software, 2000.

- Craig J, Young CA, Ennis M, et al. A randomised controlled trial comparing rehabilitation against standard therapy in multiple sclerosis patients receiving intravenous steroid treatment. I Neurol Neurosurg Psychiatry 2003;74:1225-30.

Other therapies

Currently no other immunomodulating therapies are recommended for the treatment of acute relapses. A small randomised trial of plasma exchange in patients with acute severe neurological deficits due to demyelinating diseases that had not responded to corticosteroid therapy, resulted in improvement in some patients. ${ }^{62}$ Plasma exchange may be considered in the event of a catastrophic acute relapse.

\section{Comprehensive management}

Management of acute relapses should not just be limited to corticosteroid therapy but should be comprehensive, tackling all aspects of the relapse. Practical supportive measures, such as the provision of care or equipment, may be essential and should not be forgotten. Symptomatic treatment for new symptoms from a relapse may sometimes be required. If a relapse is improving spontaneously or with corticosteroids the duration of symptoms may be too short to warrant symptomatic treatment. However, if symptoms are distressing or not resolving then treatment may be required. For example, trigeminal neuralgia attributable to an acute relapse may be very distressing even for a short period of time but may be alleviated with medication such as carbamazepine. Symptomatic treatments are not discussed further here but are reviewed elsewhere. ${ }^{63}$

Functional recovery from a relapse may be facilitated by multidisciplinary input from neurological rehabilitation services. This input should run in parallel with any medical treatment and depending on need may be on an outpatient or in-patient basis. A recent randomised controlled trial found a multidisciplinary rehabilitation approach to be superior to a standard ward routine in people with multiple sclerosis receiving intravenous corticosteroid therapy. ${ }^{64}$ In-patient rehabilitation has also been shown to be useful in relapsing remitting multiple sclerosis particularly in people with incomplete recovery from relapses with moderate to severe disability ${ }^{65}$

\section{CONCLUSION}

Multiple sclerosis is an unpredictable disease that may cause significant neurological deficit and disability. The diagnostic stage is an anxious time for the person and the diagnostic process should be rapid and adhere to good practice guidelines. Appropriate support and information should be provided from the time of diagnosis. Education should be an ongoing part of management with an emphasis on self management. Because of the unpredictable nature of relapses healthcare services should be flexible and responsive to meet acute needs. Management of acute relapses requires a comprehensive approach addressing medical, functional, and psychosocial aspects of the relapse. Although historically management has often been haphazard the introduction of clinical guidelines and the development of specialist clinical resources aims to standardise and optimise management across the United Kingdom.

\section{Authors' affiliations}

S M Leary, B Porter, A J Thompson, Rehabilitation Group, Institute of Neurology, London, UK

Funding: none.

Competing interests: none declared.

\section{REFERENCES}

1 National Institute for Clinical Excellence. Management of multiple sclerosis in primary and secondary care. London: NICE, 2003

2 Federation of Royal Colleges of Physicians of the UK. Good medical practice for physicians. London: RCP, 2004.

3 Sadovnick AD, Ebers GC, Wilson RW, et al. Life expectancy in patients attending multiple sclerosis clinics. Neurology 1992;42:991-4.

4 Sadovnick $A D$, Ebers GC. Epidemiology of multiple sclerosis: a critical overview. Can J Neurol Sci 1993;20:17-29.

5 Dean G, Kurtzke JF. On the risk of multiple sclerosis according to the age at immigration to South Africa. BMJ 1971;iii:725-9.

6 Lublin FD, Reingold SC. Defining the clinical course of multiple sclerosis: results of an international survey. National Multiple Sclerosis Society (USA) Advisory Committee on Clinical Trials of New Agents in Multiple Sclerosis. Neurology 1996;46:907-11.

7 Lublin FD, Baier M, Cutter G. Effect of relapses on development of residual deficit in multiple sclerosis. Neurology 2003;61:1528-32.

8 Weinshenker BG, Bass B, Rice GPA. The natural history of multiple sclerosis: a geographically based study. 1. Clinical course and disability. Brain 1989;112:133-46

9 Thompson AJ, Polman $\mathrm{CH}$, Miller DH, et al. Primary progressive multiple sclerosis. Brain 1997;120:1085-96.

10 Runmarker B, Andersen O. Prognostic factors in a multiple sclerosis incidence cohort with twenty-five years of follow-up. Brain 1993;116:117-34.

11 Kremenchutzky M, Cottrell D, Rice G. The natural history of multiple sclerosis: a geographically based study. 7. Progressive-relapsing and relapsingprogressive multiple sclerosis: a re-evaluation, Brain 1999:122:1941-50.

12 Andersson PB, Waubant E, Gee L, et al. Multiple sclerosis that is progressive from the time of onset: clinical characteristics and progression of disability. Arch Neurol 1999;56:1138-42.

13 Thompson AJ, Hutchinson M, Brazil J, et al. A clinical and laboratory study of benign multiple sclerosis. Q J Med 1986;58:69-80.

14 Pittock SJ, McClelland RL, Mayr WT, et al. Clinical implications of benign multiple sclerosis: a 20-year population-based follow-up study. Ann Neurol 2004;56:303-6.

15 Schumacher GA, Beebe G, Kibler RF, et al. Problems of experimental trials of therapy in multiple sclerosis: report by the panel on the evaluation of experimental trials of therapy in multiple sclerosis. Ann N Y Acad Sci 1965; 122:552-68.

16 Poser CM, Paty DW, Scheinberg L, et al. New diagnostic criteria for multiple sclerosis: Guidelines for research protocols. Ann Neurol 1983;13:227-31.

17 McDonald WI, Compston A, Edan G, et al. Recommended diagnostic criteria for multiple sclerosis: guidelines from the international panel on the diagnosis of multiple sclerosis. Ann Neurol 2001;50:121-7.

18 McDonnell GV, Hawkins SA. Clinical study of primary progressive multiple sclerosis in Northern Ireland, UK. J Neurol Neurosurg Psychiatry 1998;64:451-4.

19 Stevenson VL, Miller DH, Rovaris M, et al. Primary progressive multiple sclerosis: a clinical and MRI cross sectional study. Neurology 1999:52:839-45.

20 Thompson AJ, Montalban X, Barkhof F, et al. Diagnostic criteria for primary progressive multiple sclerosis: a position paper. Ann Neurol 2000;47:831-5.

21 Garg RK. Acute disseminated encephalomyelitis. Postgrad Med J 2003;79:11-17.

22 Matthews B. Differential diagnosis of multiple sclerosis and related disorders. In: Compston A, Ebers G, Lassmann H, et al, eds. McAlpine's multiple sclerosis. 3rd ed. London: Churchill Livingstone, 1998:223-50.

23 Kesselring J, Miller DH, Robb SA, et al. Acute disseminated encephalomyelitis. MRI findings and the distinction from multiple sclerosis. Brain 1990;113:291-302.

24 Elian M, Dean G. To tell or not to tell the diagnosis of multiple sclerosis. Lancet 1985;ii:27-8. 
25 Koopman W, Schweitzer A. The journey to multiple sclerosis: a qualitative study. J Neurosci Nurs 1999;31:17-26.

26 Robinson I. The context and consequences of communicating the diagnosis of multiple sclerosis: some brief findings from a survey of 900 patients. In: Wietholder H, Dickgans J, Merten J, eds. Current concepts in multiple sclerosis. New York: Elsevier, 1991:17-22.

27 Robinson I. The views of people with MS about their needs. Birmingham: Brunel Research Unit, 1996.

28 Freeman J, Johnson J, Rollinson S, et al. Standards of healthcare for people with multiple sclerosis. London: Multiple Sclerosis Society of Great Britain and Northern Ireland and The National Hospital for Neurology and Neurosurgery, 1997.

29 Porter B, Keenan E, Record E, et al. Diagnosis of MS: a comparison of three different clinical settings. Mult Scler 2003:9:431-9.

30 Stewart MA. Effective physician-patient communication and health outcomes: a review. Can Med Assoc J 1995;152:1423-33.

31 MS Trust, UKMSSNA, RCN. Specialist nursing in MS-the way forward: the key elements for developing MS specialist nurse services in the UK. Letchworth: MS Trust, 2001

32 Multiple Sclerosis Society. Helping you explain MS: a teaching resource for healthcare professionals. London: MSS, 2004

33 Medicines Partnership. Multiple sclerosis decisions. An independent aid to your decision. http://www.msdecisions.org.uk.

34 Lorig KR, Holman H. Self-management education: history, definition, outcomes, and mechanisms. Ann Behav Med 2003;26:1-7.

35 Kieseier BC, Hartung HP. Current disease-modifying therapies in multiple sclerosis. Semin Neurol 2003;23:133-46.

36 Khan O, Zabad R, Caon C, et al. Comparative assessment of immunomodulating therapies for relapsing-remitting multiple sclerosis. CNS Drugs 2002; 16:563-78.

37 Sibley WA, Bamford CR, Clark K. Clinical viral infections and multiple sclerosis. Lancet 1985;i:1313-15.

38 Andersen $\mathrm{O}$, Lygner PE, Bergstrom T, et al. Viral infections trigger multiple sclerosis relapses: a prospective seroepidemiological study. J Neurol 1993;240:417-22.

39 Buljevac D, Flach HZ, Hop WCJ, et al. Prospective study on the relationship between infections and multiple sclerosis exacerbations. Brain 2002; 125:952-60.

40 Miller AE, Morgante LA, Buchwald LY, et al. A multicentre, randomised double-blind, placebo controlled trial of influenza immunisation in multiple sclerosis. Neurology 1997;48:312-14.

41 Confavreux C, Suissa S, Saddier P, et al. Vaccinations and the risk of relapse in multiple sclerosis. Vaccines in Multiple Sclerosis Study Group. N Engl J Med 2001;344:319-26.

42 Rutschmann OT, McCrory DC, Matchar DB, Immunization Panel of the Multiple Sclerosis Council for Clinical Practice Guidelines. Immunization and MS: a summary of published evidence and recommendations. Neurology 2002;59:1837-43.

43 Goodin DS, Ebers GC, Johnson KP, et al. The relationship of MS to physical trauma and psychological stress. Report of the Therapeutic and Technology Assessment Subcommittee of the American Academy of Neurology. Neurology 1999;52:1737-45.

44 Mohr DC, Goodkin DE, Bacchetti P, et al. Psychological stress and the subsequent appearance of new brain lesions in MS. Neurology 2000;55:55-61.

45 Buljevac D, Hop WC, Reedeker W, et al. Self reported stressful life events and exacerbations in multiple sclerosis: prospective study. BMJ 2003;327:646.
46 Mohr DC, Hart SL, Julian L, et al. Association between stressful life events and exacerbation in multiple sclerosis: a meta-analysis. BMJ 2004;328:731

47 Confavreux C, Hutchinson M, Hours MM, et al. Rate of pregnancy-related relapse in multiple sclerosis. Pregnancy in Multiple Sclerosis Group. N Engl J Med 1998;339:285-91.

48 Gold R, Buttgereit F, Toyka KV. Mechanism of action of glucocorticosteroid hormones: possible implications for therapy of neuroimmunological disorders. J Neuroimmunol 2001;117:1-8.

49 Buckley C, Kennard C, Swash M. Treatment of acute exacerbations of multiple sclerosis with intravenous methyl-prednisolone. J Neurol Neurosurg Psychiatry 1982;45:179-80

50 Milligan NM, Newcombe R, Compston DA. A double-blind controlled trial of high dose methylprednisolone in patients with multiple sclerosis: 1. Clinical effects. J Neurol Neurosurg Psychiatry 1987;50:511-16.

51 Barnes MP, Bateman DE, Cleland PG, et al. Intravenous methylprednisolone for multiple sclerosis in relapse. J Neurol Neurosurg Psychiatry 1985:48:157-9.

52 Thompson AJ, Kennard C, Swash M, et al. Relative efficacy of intravenous methylprednisolone and ACTH in the treatment of acute relapse in MS. Neurology 1989;39:969-71.

53 Beck RW, Cleary PA, Trobe JD, et al. The effect of corticosteroids for acute optic neuritis on the subsequent development of multiple sclerosis. N Engl J Med 1993;329:1764-9.

54 Beck RW. The optic neuritis treatment trial: three-year follow-up results. Arch Ophthalmol 1995;113:136-7.

55 Alam SM, Kyriakides T, Lawden M, et al. Methylprednisolone in multiple sclerosis: a comparison of oral with intravenous therapy at equivalent high dose. J Neurol Neurosurg Psychiatry 1993;56:1219-20.

56 Barnes D, Hughes RA, Morris RW, et al. Randomised trial of oral and intravenous methylprednisolone in acute relapse of multiple sclerosis. Lancet 1997:349:902-6.

57 Tremlett HL, Luscombe DK, Wiles CM. Use of corticosteroids in multiple sclerosis by consultant neurologists in the United Kingdom. J Neurol Neurosurg Psychiatry 1998:65:362-5.

58 Filippini G, Brusaferri F, Sibley WA, et al. Corticosteroids for acute exacerbations in multiple sclerosis. Cochrane Library. Issue 4. Oxford: Update Software, 2000

59 Robson LS, Bain C, Beck S, et al. Cost analysis of methylprednisolone treatment of multiple sclerosis patients. Can J Neurol Sci 1998;25:222-9.

60 Riazi A, Hobart JC, Porter B, et al. Developing a measure of patients' experiences of relapse management in multiple sclerosis. (Abstract). Mult Scler 2003;9(suppl 1):S152.

61 Lyons PR, Newman PK, Saunders M. Methylprednisolone therapy in multiple sclerosis: a profile of adverse effects. J Neurol Neurosurg Psychiatry 1988;51:285-7.

62 Weinshenker BG, O'Brien PC, Petterson TM, et al. A randomized trial of plasma exchange in acute central nervous system inflammatory demyelinating disease. Ann Neurol 1999;46:878-86.

63 Thompson AJ. Symptomatic management and rehabilitation in multiple sclerosis. J Neurol Neurosurg Psychiatry 2001;71(suppl 2):22-7.

64 Craig J, Young CA, Ennis $M$, et al. A randomised controlled trial comparing rehabilitation against standard therapy in multiple sclerosis patients receiving intravenous steroid treatment. J Neurol Neurosurg Psychiatry 2003;74:1225-30

65 Liu C, Playford ED, Thompson AJ. Does neurorehabilitation have a role in relapsing-remitting multiple sclerosis. J Neurol 2003;250:1214-18. 